\title{
Lifshitz theory of van der Waals pressure in dissipative media
}

\author{
Yi Zheng and Arvind Narayanaswamy* \\ Department of Mechanical Engineering, Columbia University, New York, New York 10027, USA
}

(Received 27 November 2010; published 11 April 2011)

\begin{abstract}
We derive a first-principles method of determining the van der Waals or Casimir pressure in a dissipative and dispersive planar multilayered system by calculating the Maxwell stress tensor in a fictitious layer of vacuum, that is eventually made to vanish, introduced in the structure. This is illustrated by calculating the van der Waals pressure in a thin film with dissipative properties embedded between two semi-infinite media.
\end{abstract}

DOI: 10.1103/PhysRevA.83.042504

PACS number(s): 31.30.jh, 03.50.De, 12.20.-m, 42.50.Ct

\section{INTRODUCTION}

van der Waals (vdW) forces, resulting from alteration of the quantum and thermal fluctuations of the electrodynamic field due to the presence of interfaces, play a significant role in the interactions between macroscopic objects at micrometerand nanometer-length scales. Hamaker was the first to extend the concept of London-vdW forces between two atoms to forces between macroscopic spheres by pairwise summation of the interaction energy between the atoms that constitute the spheres [1]. Lifshitz, in his seminal work [2], outlined a method based on Rytov's theory of fluctuational electrodynamics [3] for computing the vdW forces between two semi-infinite regions separated by a vacuum gap. It required calculation of the average value of the Maxwell stress tensor in the vacuum gap. The generalization of Lifshitz's method to calculating $\mathrm{vdW}$ forces between semi-infinite regions separated by dissipative media is not straightforward because of the difficulty in defining an electromagnetic stress tensor in dissipative media [4]. In this paper we show that it is possible to generalize Lifshitz's theory to determine the vdW pressure in arbitrary planar media with dissipative and dispersive electromagnetic properties without having to define the electromagnetic stress tensor or free energy in any material but vacuum.

An approach proposed by Dzyaloshinskii, Lifshitz, and Pitaevskii (DLP from now on) [5], cast in the language of quantum field theory, is the most frequently used generalization of Lifshitz's method to calculate forces between objects separated by absorbing media. Even though it has been noted that an expression for the Maxwell stress tensor for timevarying fields in absorbing media cannot be expressed in terms of the frequency-dependent permittivity and permeability alone [4], the DLP method effectively reduces to using a "Minkowski-like" [6] definition of the electromagnetic stress tensor in dissipative media. Ninham et al. [7] circumvented the complications of the DLP method but, in doing so, had to postulate that the free energy of an electromagnetic mode at frequency $\omega_{j}$ is given by $k_{B} T \ln \left[\sinh \left(\hbar \omega_{j} / 2 k_{B} T\right)\right]$, where $k_{B}$ is Boltzmann's constant, $2 \pi \hbar$ is Planck's constant, and $T$ is the absolute temperature, even though the mode frequencies in dissipative media are, in general, complex. It has been argued by Barash and Ginzburg [8,9] that ascribing to each mode a free energy of the above-mentioned form is indeed correct. The methods of DLP and Barash and Ginzburg are justified on the

*arvind.narayanaswamy@ columbia.edu grounds that it is possible to ascribe thermodynamic functions to electromagnetic fields in equilibrium with matter $[6,9]$. While many authors have attempted to generalize Lifshitz theory to determining $\mathrm{vdW}$ pressure in dissipative media, they do so by assuming an expression for the electrodynamic stress tensor [10] or by defining a Lagrangian density for the electrodynamic field [11], both of which are debatable for media with dissipation [4].

The relative transparency of the Lifshitz method is obscured by the complexity of Dzyaloshinskii's formalism or by having to define the free energy of each mode, even though the final result is a simple generalization of the Lifshitz formula. It has been generally regarded that Lifshitz's method, in which the stress tensor definition is above reproach, is incapable of handling dissipative media without relying on either of the two generalizations [6]. Using the fluctuation-dissipation theorem and properties of the dyadic Green's function, we express the components of the Maxwell stress tensor in vacuum in terms of components of the dyadic Green's function [12]. After a description of a general method to deal with multilayered media, we show, using examples of (1) a thin film bound by vacuum on both sides, (2) a thin film with vacuum on one side and a semi-infinite medium with arbitrary permittivity and permeability on the other, and (3) a thin film bound by semiinfinite media with arbitrary permittivity and permeability, that the expression for vdW pressure coincides with that of DLP.

\section{GENERAL FORMULATION OF VAN DER WAALS ENERGY AND PRESSURE IN MULTILAYERED MEDIA}

Let us analyze a general multilayer system, as shown in Fig. 1(a), and express the vdW free energy of the system in terms of combinations of vdW free energy of smaller units. The vdW free energy per unit area of a planar configuration of $N$ layers [Fig. 1(a)] sandwiched between two semi-infinite media, medium $L$ to the left and medium $R$ to the right, is represented by $U_{L R}\left(z_{1}, \ldots, z_{N}\right)$. Each layer is characterized by not only the thickness $z_{k}$ but also the permittivity $\varepsilon_{k}$ and permeability $\mu_{k}$ (both relative to that of vacuum). We use the aforementioned notation for free energy for its efficiency. If one of the semi-infinite media is vacuum, the subscript $V$ is used instead of $L$ or $R$. $U_{L R}\left(z_{1}, \ldots, z_{N}\right)$ can be written as a combination of three terms: (1) the free energy of the first $k$ layers sandwiched by semi-infinite medium $L$ to the left and vacuum to the right of the $k$ th layer, $U_{L V}\left(z_{1}, \ldots, z_{k}\right)$, and (2) the free energy of the remaining $N-k$ layers sandwiched by 


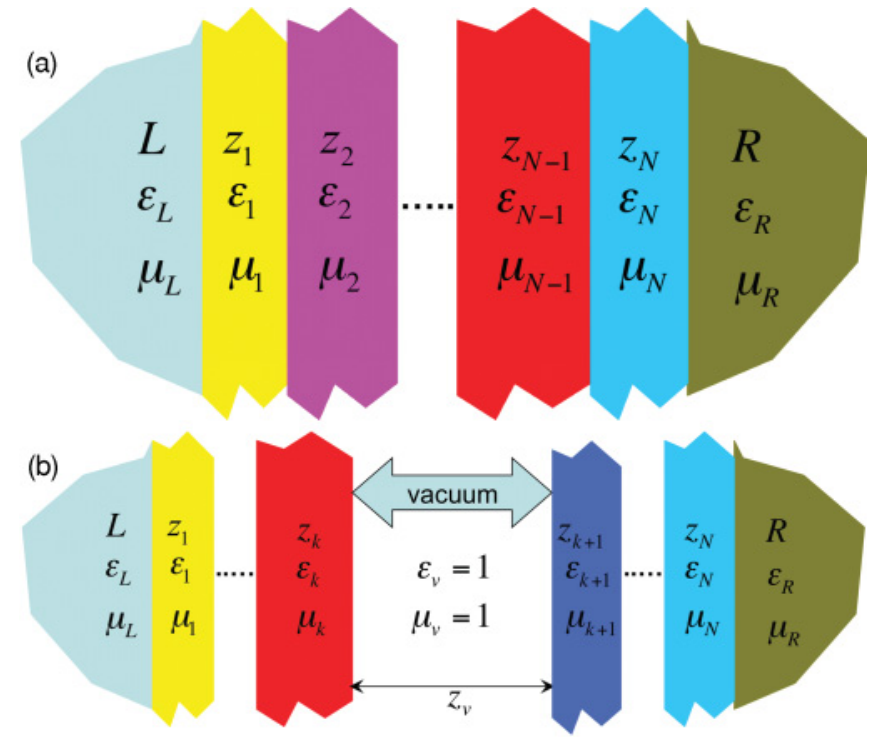

FIG. 1. (Color online) (a) A multilayer system with $N$ layers between two semi-infinite regions $L$ and $R$. (b) Method of splitting the $N$ layer multilayer system into components. The $z$ axis is perpendicular to the interfaces.

semi-infinite medium $R$ to the right and vacuum to the left of the $(k+1)$ th layer, $U_{V R}\left(z_{k+1}, \ldots, z_{N}\right)$, and (3) the work done in bringing the two systems from infinite separation to a separation $\delta \rightarrow 0$. This statement can be written as

$$
\begin{aligned}
U_{L R}\left(z_{1}, \ldots, z_{N}\right)= & U_{L V}\left(z_{1}, \ldots, z_{k}\right)+U_{V R}\left(z_{k+1}, \ldots, z_{N}\right) \\
& +\lim _{\delta \rightarrow 0} \int_{\infty}^{\delta} T_{z z}^{\text {avg }}\left(z_{v}\right) d z_{v}
\end{aligned}
$$

where $T_{z z}^{\text {avg }}\left(z_{v}\right) \equiv T_{z z}^{\text {avg }}\left(z_{1}, \ldots, z_{k}, z_{v}, z_{k+1}, \ldots, z_{N}\right)$ is the vdW pressure in the vacuum region in Fig. 1(b) against which work needs to be done to create the $N$ layer system from the two subsystems. The partial derivative $\partial U_{L R}\left(z_{1}, \ldots, z_{N}\right) / \partial z_{r}=p_{L R}^{(r)}\left(z_{1}, \ldots, z_{N}\right)$ gives the vdW pressure in the $r$ th layer of the $N$ layer system bounded by $L$ and $R$. For a thin film bounded by two semi-infinite regions, we drop the superscript $(r)$ and denote the pressure simply as $p_{L R}$. By differentiating Eq. (1) with respect to $z_{r}$, we obtain the following equation for $p_{L R}^{(r)}$ :

$$
\begin{aligned}
p_{L R}^{(r)}\left(z_{1}, \ldots, z_{N}\right)= & \frac{\partial U_{L V}}{\partial z_{r}}\left(z_{1}, \ldots, z_{k}\right)+\frac{\partial U_{V R}}{\partial z_{r}}\left(z_{k+1}, \ldots, z_{N}\right) \\
& +\int_{\infty}^{0} \frac{\partial T_{z z}^{\mathrm{avg}}}{\partial z_{r}}\left(z_{v}\right) d z_{v} .
\end{aligned}
$$

One of the first two terms on the right-hand side of Eq. (2) is zero, depending on whether $1 \leqslant r \leqslant k$ or $k+1 \leqslant r \leqslant N$. Though $T_{z z}^{\text {avg }}\left(z_{v}\right)$ diverges as $z_{v}^{-3}$ for $z_{v} \rightarrow 0$, the quantity $\partial T_{z z}^{\text {avg }} / \partial z_{r}$ is finite as $z_{v} \rightarrow 0 \forall 1 \leqslant r \leqslant N$, allowing us to define the partial derivative of the last term in Eq. (1) as the integral $\int_{\infty}^{0} \partial T_{z z}^{\text {avg }} / \partial z_{r} d z_{v}$ (see Appendix A for justification). $T_{z z}^{\text {avg }}\left(z_{v}\right)$ is obtained simply by determining the $z z$ component of the Maxwell stress tensor in the vacuum region. Using the procedure described above, we can write the vdW free energy of any $N$ layer medium in terms of $U_{V V}\left(z_{1}\right), U_{V V}\left(z_{2}\right), \ldots$, and $U_{V V}\left(z_{N}\right)$, and contributions from terms of the form $\int_{\infty}^{\delta} T_{z z}^{\text {avg }}\left(z_{v}\right) d z_{v}$, all of which involve calculation of the Maxwell stress tensor in vacuum alone. $U_{V V}(z)$ is nothing but the vdW free energy to create a thin film of thickness $z$ in free space.

We rely on Rytov's theory of fluctuational electrodynamics to determine the value of $T_{z z}^{\text {avg }}$. The cross-spectral correlations of the electric field components can be written as $\left\langle E_{p}(\boldsymbol{r}, \omega) E_{q}^{*}(\boldsymbol{r}, \omega)\right\rangle=\left(2 \omega \mu_{o} \Theta / \pi\right) \operatorname{Im} \boldsymbol{G}_{p q}^{e}(\boldsymbol{r}, \boldsymbol{r})$, where $p, q=$ $x, y, z, \Theta=(\hbar \omega / 2) \operatorname{coth}\left(\hbar \omega / 2 k_{B} T\right)$, and $\boldsymbol{G}_{p q}^{e}$ is the $p q$ component of the electric dyadic Green's function $[12,13]$. The spectral correlation is defined such that $\left\langle E_{p}(\boldsymbol{r}, t) E_{q}(\boldsymbol{r}, t)\right\rangle=$ $\int_{0}^{\infty} d \omega\left\langle E_{p}(\boldsymbol{r}, \omega) E_{q}^{*}(\boldsymbol{r}, \omega)\right\rangle$. Similarly, the cross-spectral correlations of the magnetic field components are given by $\left\langle H_{p}(\boldsymbol{r}, \omega) H_{q}^{*}(\boldsymbol{r}, \omega)\right\rangle=\left(2 \omega \epsilon_{o} \Theta / \pi\right) \operatorname{Im} \boldsymbol{G}_{p q}^{m}(\boldsymbol{r}, \boldsymbol{r})$. The dyadic Green's functions $\boldsymbol{G}^{e}$ and $\boldsymbol{G}^{m}$ are electromagnetic duals of each other and are solutions of $\nabla \times \nabla \times \boldsymbol{G}\left(\boldsymbol{r}, \boldsymbol{r}^{\prime}\right)-$ $k^{2} \boldsymbol{G}\left(\boldsymbol{r}, \boldsymbol{r}^{\prime}\right)=\boldsymbol{I} \delta\left(\boldsymbol{r}-\boldsymbol{r}^{\prime}\right)$, where $\boldsymbol{I}$ is the identity dyad, $\boldsymbol{r}$ and $\boldsymbol{r}^{\prime}$ are the position vectors for observation and source, respectively. $\boldsymbol{G}^{e}$ and $\boldsymbol{G}^{m}$ are obtained by enforcing the continuity of (1) $\mu(\boldsymbol{r})\left[\hat{\boldsymbol{n}} \times \boldsymbol{G}^{e}\left(\boldsymbol{r}, \boldsymbol{r}^{\prime}\right)\right],(2) \hat{\boldsymbol{n}} \times \nabla \times \boldsymbol{G}^{e}\left(\boldsymbol{r}, \boldsymbol{r}^{\prime}\right)$, (3) $\varepsilon(\boldsymbol{r})\left[\hat{\boldsymbol{n}} \times \boldsymbol{G}^{m}\left(\boldsymbol{r}, \boldsymbol{r}^{\prime}\right)\right]$, and (4) $\hat{\boldsymbol{n}} \times \boldsymbol{\nabla} \times \boldsymbol{G}^{m}\left(\boldsymbol{r}, \boldsymbol{r}^{\prime}\right)$ on either side of an interface defined by the unit normal vector $\hat{\boldsymbol{n}}$ at the point $\boldsymbol{r}$.

The $z z$ component of the Maxwell stress tensor in vacuum can be expressed in terms of $\boldsymbol{G}^{\boldsymbol{e}}$ and $\boldsymbol{G}^{m}$ as $T_{z z}(\boldsymbol{r}, \omega)=$ $\left(2 \omega \Theta / \pi c^{2}\right) \operatorname{Im} G(\boldsymbol{r}, \omega)[12,13]$, where $G(\boldsymbol{r}, \omega)=\boldsymbol{G}_{z z}^{e}(\boldsymbol{r}, \boldsymbol{r})-$ $\frac{1}{2} \operatorname{Tr} \boldsymbol{G}^{e}(\boldsymbol{r}, \boldsymbol{r})+\boldsymbol{G}_{z z}^{m}(\boldsymbol{r}, \boldsymbol{r})-\frac{1}{2} \operatorname{Tr} \boldsymbol{G}^{m}(\boldsymbol{r}, \boldsymbol{r})$. The average value of the $z z$ component of the Maxwell stress tensor, $T_{z z}^{\text {avg }}$, at any instant of time at position $\boldsymbol{r}$ in a vacuum layer is given by:

$$
T_{z z}^{\mathrm{avg}}=\int_{0}^{\infty} \frac{\hbar \omega^{2}}{\pi c^{2}} \operatorname{coth}\left(\frac{\hbar \omega}{2 k_{B} T}\right) \operatorname{Im} G(\boldsymbol{r}, \omega) d \omega
$$

$\boldsymbol{G}^{e}$ and $\boldsymbol{G}^{m}$ are analytic in the upper half plane (UHP) by virtue of being response functions. Since $G(\boldsymbol{r}, \omega)$ is a linear combination of different components of $\boldsymbol{G}^{\boldsymbol{e}}$ and $\boldsymbol{G}^{m}$, it is also analytic in the UHP. We can therefore use Lifshitz's technique to replace the integral over $\omega$ along the real positive frequency axis by a summation over Matsubara frequencies on the imaginary frequency axis in the UHP as

$$
T_{z z}^{\text {avg }}=-\frac{2 k_{B} T}{c^{2}} \sum_{n=0}^{\infty}{ }^{\prime} \xi_{n}^{2} G\left(\boldsymbol{r}, i \xi_{n}\right)=k_{B} T \sum_{n=0}^{\infty}{ }^{\prime} K_{n}
$$

where, $\quad \xi_{n}=2 \pi n k_{B} T / \hbar, \quad K_{n}=-2 \xi_{n}^{2} G\left(\boldsymbol{r}, i \xi_{n}\right) / c^{2}, \quad K_{0}=$ $-\lim _{\xi \rightarrow 0} 2 \xi^{2} G(\boldsymbol{r}, i \xi) / c^{2}$, and $n=0,1,2, \ldots$. The prime $\left(^{\prime}\right)$ next to $\sum$ indicates that the $n=0$ term is given weight 0.5 . $G\left(\boldsymbol{r}, i \xi_{n}\right)$ can be written in terms of the reflection coefficients of plane waves that comprise $\boldsymbol{G}^{e}$ and $\boldsymbol{G}^{m}$ [14]. We now apply this method to calculating the vdW pressure in a thin film (indicated by $m$ ) bounded by two semi-infinite objects, $L$ and $R$. To do so, we introduce a vacuum layer, shown in Fig. 2, in which the Maxwell stress tensor will be determined.

\section{APPLICATION TO THIN FILMS}

We start with the assertion that the vdW pressure in any infinite or semi-infinite planar medium is zero. We show, using the following three examples, that the proposed method is in agreement with the predictions of DLP theory for the case of a thin film between two semi-infinite objects. $K_{n}$, from which 
$T_{z z}^{\mathrm{avg}}$ can be calculated using Eq. (4), for the configuration shown in Fig. 2 is given by

$$
K_{n}=\frac{1}{\pi} \int_{0}^{\infty} \sum_{p=e, h} \frac{R_{v L}^{(p)} \widetilde{R}_{v R}^{(p)} e^{-2 k_{z v} z_{v}}}{1-R_{v L}^{(p)} \widetilde{R}_{v R}^{(p)} e^{-2 k_{z v} z_{v}}} k_{z v} k_{\rho} d k_{\rho}
$$

where $p=e, h$ refer to the transverse electric and transverse magnetic polarizations, respectively, and

$$
\begin{aligned}
\widetilde{R}_{v R}^{(p)}=\frac{R_{v m}^{(p)}+R_{m R}^{(p)} e^{-2 k_{z m} z_{m}}}{1+R_{v m}^{(p)} R_{m R}^{(p)} e^{-2 k_{z m} z_{m}}}, \quad \text { expression for } \int_{\infty}^{0} \frac{\partial K_{n}}{\partial z_{m}} d z_{v}, \\
\int_{\infty}^{0} \frac{\partial K_{n}}{\partial z_{m}} d z_{v}=\frac{1}{\pi} \int_{0}^{\infty} \sum_{p=e, h} \frac{R_{v L}^{(p)} R_{m R}^{(p)}\left(1-R_{v m}^{(p) 2}\right) e^{-2 k_{z m} z_{m}}}{\left[\left(1-R_{v L}^{(p)} R_{v m}^{(p)}\right)-\left(R_{v L}^{(p)} R_{m R}^{(p)}+R_{m v}^{(p)} R_{m R}^{(p)}\right) e^{-2 k_{z m} z_{m}}\right]\left(1+R_{v m}^{(p)} R_{m R}^{(p)} e^{\left.-2 k_{z m} z_{m}\right)}\right.} k_{z m} k_{\rho} d k_{\rho}
\end{aligned}
$$

$$
\begin{gathered}
R_{v L}^{(e)}=\frac{k_{z v} \mu_{L}-k_{z L} \mu_{v}}{k_{z v} \mu_{L}+k_{z L} \mu_{v}}, \quad R_{v L}^{(h)}=\frac{k_{z v} \varepsilon_{L}-k_{z L} \varepsilon_{v}}{k_{z v} \varepsilon_{L}+k_{z L} \varepsilon_{v}}, \\
k_{z v}=\sqrt{k_{\rho}^{2}+\varepsilon_{v} \mu_{v} \xi_{n}^{2} / c^{2}}, \quad k_{z m}=\sqrt{k_{\rho}^{2}+\varepsilon_{m} \mu_{m} \xi_{n}^{2} / c^{2}},
\end{gathered}
$$

and similarly for reflection coefficients at other interfaces and wave vectors in other layers. All permittivities and permeabilities are evaluated at $i \xi_{n}, n=0,1,2, \ldots$ For reflection coefficients, the subscript $v$ is used to denote an interface with vacuum. Since it is $\frac{\partial}{\partial z_{m}} \int_{\infty}^{0} T_{z z}^{\text {avg }}\left(z_{m}, z_{v}\right) d z_{v}$ that will eventually be used in calculating vdW pressure, below we give the
Example 1: Vacuum-Thin Film-Vacuum. To find the vdW pressure in a thin film of material of thickness $z_{m}$, we consider a four-layer configuration, as shown in Fig. 2, with $L$ being replaced with material $m$, and $R$ being vacuum. If the vdW energy for creating a film of thickness $z_{m}$ is $U_{V V}\left(z_{m}\right)$, the following equation can be written for conservation of energy for moving the thin film from $z_{v}=\infty$ to $z_{v}=\delta \rightarrow 0$ :

$$
U_{V V}\left(z_{m}\right)+\lim _{\delta \rightarrow 0} \int_{\infty}^{\delta} T_{z z}^{\mathrm{avg}} d z_{v}=U_{o}
$$

where $U_{o}$ is an arbitrary constant that is the energy per unit area of a semi-infinite medium $M$ adjacent to a semi-infinite region of vacuum. Differentiation of Eq. (8) with respect to $z_{m}$ gives the following equation for vdW pressure:

$$
p_{V V}\left(z_{m}\right)+\int_{\infty}^{0} \frac{\partial T_{z z}^{\mathrm{avg}}}{\partial z_{m}} d z_{v}=0 .
$$

Using Eqs. (4), (7), and (9), the vdW pressure in a thin film of medium $m$ bounded by vacuum is given by [see Eq. (B1) in Appendix B for further details]

$p_{V V}\left(z_{m}\right)=\frac{k_{B} T}{\pi} \sum_{n=0}^{\infty} \int_{0}^{\infty} \sum_{p=e, h} \frac{R_{m v}^{(p) 2} e^{-2 k_{z m} z_{m}}}{1-R_{m v}^{(p) 2} e^{-2 k_{z m} z_{m}}} k_{z m} k_{\rho} d k_{\rho}$,

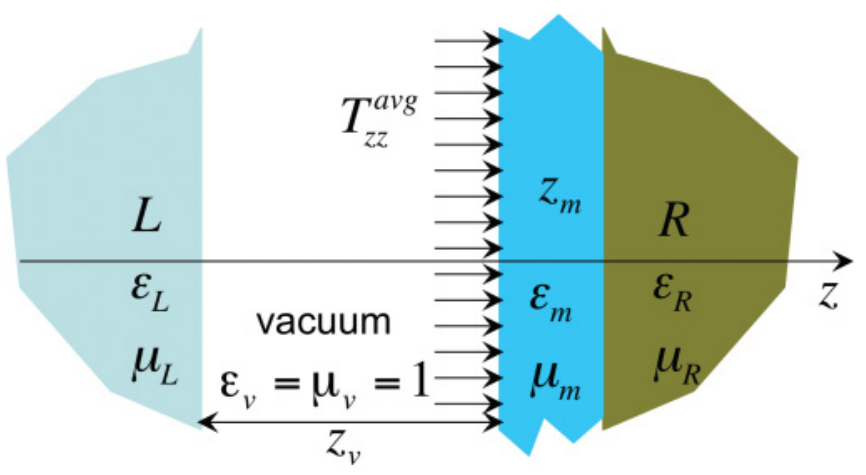

FIG. 2. (Color online) A four-layer system. where each integral is evaluated at the Matsubara frequency $\xi_{n}=2 \pi n k_{B} T / \hbar$.

Example 2: Material-Thin Film-Vacuum. To find $p_{L V}\left(z_{m}\right)$, we consider a four-layer configuration, as shown in Fig. 2, with $R$ being vacuum. Equation (1) can be modified for the four-layer system to give the following equation for $U_{L V}\left(z_{m}\right)$ :

$$
U_{L V}\left(z_{m}\right)=U_{V V}\left(z_{m}\right)+\lim _{\delta \rightarrow 0} \int_{\infty}^{\delta} T_{z z}^{\mathrm{avg}} d z_{v}
$$

Differentiating Eq. (11) with respect to $z_{m}$, we obtain the following equation for $p_{L V}\left(z_{m}\right)$ in terms of $p_{V V}\left(z_{m}\right)$, which has been calculated earlier, and $T_{z z}^{\text {avg }}$ :

$$
p_{L V}\left(z_{m}\right)=p_{V V}\left(z_{m}\right)+\int_{\infty}^{0} \frac{\partial T_{z z}^{\mathrm{avg}}}{\partial z_{m}} d z_{v}
$$

Using the expressions for $p_{V V}$ [Eq. (10)] and Eq. (7), we obtain the following equation for $p_{L V}\left(z_{m}\right)$ [see Eq. (B2) in Appendix B for further details]:

$$
\begin{aligned}
p_{L V}\left(z_{m}\right)= & \frac{k_{B} T}{\pi} \\
& \times \sum_{n=0}^{\infty}, \int_{0}^{\infty} \sum_{p=e, h} \frac{R_{m L}^{(p)} R_{m v}^{(p)} e^{-2 k_{z m} z_{m}}}{1-R_{m L}^{(p)} R_{m v}^{(p)} e^{-2 k_{z m} z_{m}}} k_{z m} k_{\rho} d k_{\rho} .
\end{aligned}
$$

We can obtain the vdW pressure $p_{V R}\left(z_{m}\right)$ by replacing $L$ with $R$ in Eq. (13).

Example 3: Material-Thin Film-Material. The vdW free energy of the system $L-m-R$ is obtained by adding to the free energy $U_{V R}\left(z_{m}\right)$ the work done in moving this system from infinite separation to the surface of a semi-infinite region of material $L$. Written as an equation, we get

$$
U_{L R}\left(z_{m}\right)=U_{V R}\left(z_{m}\right)+\lim _{\delta \rightarrow 0} \int_{\infty}^{\delta} T_{z z}^{\mathrm{avg}} d z_{v}
$$


and $p_{L R}\left(z_{m}\right)$ is given by [see Eq. (B4) in Appendix B for further details]

$$
\begin{aligned}
p_{L R}\left(z_{m}\right)= & p_{V R}\left(z_{m}\right)+\int_{\infty}^{0} \frac{\partial T_{z z}^{\mathrm{avg}}}{\partial z_{m}} d z_{v}=\frac{k_{B} T}{\pi} \\
& \times \sum_{n=0}^{\infty}, \int_{0}^{\infty} \sum_{p=e, h} \frac{R_{m L}^{(p)} R_{m R}^{(p)} e^{-2 k_{z m} z_{m}}}{1-R_{m L}^{(p)} R_{m R}^{(p)} e^{-2 k_{z m} z_{m}}} k_{z m} k_{\rho} d k_{\rho}
\end{aligned}
$$

In writing Eqs. (11) and (14), we have omitted a term similar to $U_{o}$ in Eq. (8) because it is contained in $U_{L V}\left(z_{m}\right)$ and $U_{L R}\left(z_{m}\right)$, respectively. It can be seen that Eq. (15) for $p_{L R}\left(z_{m}\right)$ is a generalization of Eqs. (10) and (13). Further simplification of Eq. (15), as shown in Appendix C, results in the following expression for $p_{L R}$ :

$$
\begin{aligned}
p_{L R}\left(z_{m}\right)= & \frac{k_{B} T}{\pi c^{3}} \sum_{n=0}^{\infty} '\left(\varepsilon_{m} \mu_{m}\right)^{3 / 2} \xi_{n}^{3} \int_{1}^{\infty} d q q^{2} \\
& \times \sum_{p=e, h}\left(R_{m L}^{(p)-1} R_{m R}^{(p)-1} e^{2 q \xi_{n} \sqrt{\varepsilon_{m} \mu_{m}} z_{m} / c}-1\right)^{-1}
\end{aligned}
$$

where $q=k_{z m} /\left(\xi_{n} \sqrt{\varepsilon_{m}\left(i \xi_{n}\right) \mu_{m}\left(i \xi_{n}\right)} / c\right)$. Equation (16) agrees with the expression for vdW pressure in a thin film according to DLP $[5,15]$. We stress that the method outlined here for calculating vdW pressure is valid irrespective of computation of the electromagnetic stress tensor by a summation along the imaginary frequency axis or along the real frequency axis. The extension to a multilayered medium is simply an exercise in determining the appropriate reflection and transmission coefficients $[14,16]$.

We have provided here a transparent formalism for calculating vdW or Casimir pressure in dissipative and dispersive media that are constituents of planar multilayer structures without having to define or calculate the stress tensor in such layers. We provide evidence backing the generalization of Lifshitz theory of vdW forces without relying on quantum field theoretic techniques employed by Dzyaloshinskii, Lifshitz, and Pitaevskii. These results offer further proof of the validity of the Minkowski-like stress tensor for calculating vdW forces, at least in planar multilayered media.

\section{ACKNOWLEDGMENTS}

The authors would like to acknowledge correspondence and discussions with Prof. Adrian Parsegian, Prof. Rudi Podgornik, and Prof. L. Pitaevskii. We also thank Prof. Pitaevskii for bringing to our attention that Dzyaloshinskii, Lifshitz, and Pitaevskii had started to solve the problem of vdW pressure in dissipative media by the method outlined in this paper but put an end to it on the suggestion of Landau [17]! This work has been funded partially by the National Science Foundation through Grant CBET-0853723.

\section{APPENDIX A: JUSTIFICATION FOR EXISTENCE OF $\int_{\infty}^{0} \partial T_{z z}^{\mathrm{avg}} / \partial z_{r} d z_{v}$}

Using Eq. (4) in the manuscript, we can see that the $z z$ component of the Maxwell stress tensor in the vacuum layer in Fig. 1(b) (in manuscript) is given by

$$
\begin{aligned}
T_{z z}^{\mathrm{avg}}\left(z_{v}\right)= & \frac{k_{B} T}{\pi} \sum_{n=0}^{\infty}, \int_{0}^{\infty} d k_{\rho} k_{z v} k_{\rho} \\
& \times \sum_{p=e, h} \frac{\widetilde{R}_{v L}^{(p)} \widetilde{R}_{v R}^{(p)} e^{-2 k_{z v} z_{v}}}{1-\widetilde{R}_{v L}^{(p)} \widetilde{R}_{v R}^{(p)} e^{-2 k_{z v} z_{v}}}
\end{aligned}
$$

where $\widetilde{R}_{v L}$ and $\widetilde{R}_{v R}$ are generalized reflection coefficients that can be determined from the boundary conditions at each interface, $\widetilde{R}_{v L} \equiv \widetilde{R}_{v L}\left(z_{1}, \ldots, z_{k}\right)$ and $\widetilde{R}_{v R} \equiv \widetilde{R}_{v R}\left(z_{k+1}, \ldots, z_{N}\right)$. The work done in displacing the multilayer stack adjoining $R$ from $z_{v}=\infty$ to $z_{v}=\delta$ is given by

$$
\begin{aligned}
\int_{\infty}^{\delta} T_{z z}^{\mathrm{avg}}\left(z_{v}\right) d z_{v}= & \frac{k_{B} T}{2 \pi} \sum_{n=0}^{\infty} \int_{0}^{\infty} d k_{\rho} k_{\rho} \\
& \times \sum_{p=e, h} \ln \left(1-\widetilde{R}_{v L}^{(p)} \widetilde{R}_{v R}^{(p)} e^{-2 k_{z v} \delta}\right)
\end{aligned}
$$

$$
\begin{aligned}
\Rightarrow & \frac{\partial}{\partial z_{r}} \lim _{\delta \rightarrow 0} \int_{\infty}^{\delta} T_{z z}^{\mathrm{avg}}\left(z_{v}\right) d z_{v} \\
= & -\frac{k_{B} T}{2 \pi} \sum_{n=0}^{\infty} \lim _{\delta \rightarrow 0} \int_{0}^{\infty} d k_{\rho} k_{\rho} \\
& \times \sum_{p=e, h} \frac{\frac{\partial}{\partial z_{r}}\left(\widetilde{R}_{v L}^{(p)} \widetilde{R}_{v R}^{(p)}\right) e^{-2 k_{z v} \delta}}{1-\widetilde{R}_{v L}^{(p)} \widetilde{R}_{v R}^{(p)} e^{-2 k_{z v} \delta}}
\end{aligned}
$$

where $\frac{\partial}{\partial z_{r}}\left(\widetilde{R}_{v L}^{(p)} \widetilde{R}_{v R}^{(p)}\right)=\widetilde{R}_{v R}^{(p)} \frac{\partial \widetilde{R}_{v L}^{(p)}}{\partial z_{r}}$ or $\widetilde{R}_{v L}^{(p)} \frac{\partial \widetilde{R}_{v R}^{(p)}}{\partial z_{r}}$, depending on whether $1 \leqslant r \leqslant k$ or $k+1 \leqslant r \leqslant N$. The product $\widetilde{R}_{v L}^{(p)} \widetilde{R}_{v R}^{(p)}$ can be written in the form $\frac{A_{1}+A_{2} e^{-2 k_{z r} z_{r}}}{A_{3}+A_{4} e^{-2 k_{z r} z_{r}}}$, where $A_{1}, A_{2}, A_{3}$, and $A_{4}$ are not functions of $z_{r}$. So the partial differentiation $\frac{\partial}{\partial z_{r}}$ yields a function of the form $-2 k_{z r} \frac{\left(A_{3} A_{2}-A_{1} A_{4}\right)}{\left(A_{3}+A_{4} e^{-2 k_{z r} z_{r}}\right)^{2}} e^{-2 k_{z r} z_{r}}$. In general, we can write

$$
\frac{\partial}{\partial z_{r}}\left(\widetilde{R}_{v L}^{(p)} \widetilde{R}_{v R}^{(p)}\right)=-2 k_{z r} \frac{N}{D} e^{-2 k_{z r} z_{r}}
$$

where $N \equiv A_{3} A_{2}-A_{1} A_{4}$ and $D \equiv\left(A_{3}+A_{4} e^{-2 k_{z r} z_{r}}\right)^{2}$. Substituting the above relation in Eq. (A3), we get

$$
\begin{aligned}
& \frac{\partial}{\partial z_{r}} \lim _{\delta \rightarrow 0} \int_{\infty}^{\delta} T_{z z}^{\mathrm{avg}}\left(z_{v}\right) d z_{v} \\
& \quad=\frac{k_{B} T}{\pi} \sum_{n=0}^{\infty} \lim _{\delta \rightarrow 0} \int_{0}^{\infty} d k_{\rho} k_{\rho} k_{z r} \sum_{p=e, h} \frac{\frac{N}{D} e^{-2 k_{z r} z_{r}} e^{-2 k_{z v} \delta}}{1-\widetilde{R}_{v L}^{(p)} \widetilde{R}_{v R}^{(p)} e^{-2 k_{z v} \delta}} .
\end{aligned}
$$


Because of the presence of the $e^{-2 k_{z r} z_{r}}$, we are justified in putting $\delta=0$ to give

$$
\begin{aligned}
& \frac{\partial}{\partial z_{r}} \lim _{\delta \rightarrow 0} \int_{\infty}^{\delta} T_{z z}^{\mathrm{avg}}\left(z_{v}\right) d z_{v} \\
& \quad=\frac{k_{B} T}{\pi} \sum_{n=0}^{\infty} \int_{0}^{\infty} d k_{\rho} k_{\rho} k_{z r} \sum_{p=e, h} \frac{\frac{N}{D} e^{-2 k_{z r} z_{r}}}{1-\widetilde{R}_{v L}^{(p)} \widetilde{R}_{v R}^{(p)}} \\
& \quad=\int_{\infty}^{0} \frac{\partial T_{z z}^{\mathrm{avg}}}{\partial z_{r}} d z_{v} .
\end{aligned}
$$

For the examples in our manuscripts, $\widetilde{R}_{v L}^{(p)}=R_{v L}^{(p)}, \widetilde{R}_{v R}^{(p)}=$ $\frac{R_{v m}^{(p)}+R_{m R}^{(p)} e^{-2 k_{z m} z_{m}}}{1+R_{v m}^{(p)} R_{m R}^{(p)} e^{-2 k_{z m} z_{m}}}, N=R_{v L}^{(p)} R_{m R}^{(p)}\left(1-R_{v m}^{(p) 2}\right)$, and $D=$ $\left(1+R_{v m}^{(p)} R_{m R}^{(p)} e^{-2 k_{z m} z_{m}}\right)^{2}$.

APPENDIX B: DERIVATION FOR EQS. (10), (13), and (15)

$$
\begin{aligned}
& p_{V V}\left(z_{m}\right)=-\int_{\infty}^{0} \frac{\partial T_{z z}^{\mathrm{avg}}}{\partial z_{m}} d z_{v}=-k_{B} T \sum_{n=0}^{\infty} \int_{\infty}^{0} \frac{\partial K_{n}}{\partial z_{m}} d z_{v} \\
& =-\frac{k_{B} T}{\pi} \sum_{n=0}^{\infty} \int_{0}^{\infty} \sum_{p=e, h} \frac{R_{v m}^{(p)} R_{m v}^{(p)}\left(1-R_{v m}^{(p) 2}\right) e^{-2 k_{z m} z_{m}}}{\left[\left(1-R_{v m}^{(p)} R_{v m}^{(p)}\right)-\left(R_{v m}^{(p)} R_{m v}^{(p)}+R_{m v}^{(p)} R_{m v}^{(p)}\right) e^{-2 k_{z m} z_{m}}\right]\left(1+R_{v m}^{(p)} R_{m v}^{(p)} e^{-2 k_{z m} z_{m}}\right)} k_{z m} k_{\rho} d k_{\rho} \\
& =\frac{k_{B} T}{\pi} \sum_{n=0}^{\infty} \int_{0}^{\infty} \sum_{p=e, h} \frac{R_{m v}^{(p) 2} e^{-2 k_{z m} z_{m}}}{1-R_{m v}^{(p) 2} e^{-2 k_{z m} z_{m}}} k_{z m} k_{\rho} d k_{\rho} \\
& p_{L V}\left(z_{m}\right)=p_{V V}\left(z_{m}\right)+\int_{\infty}^{0} \frac{\partial T_{z z}^{\mathrm{avg}}}{\partial z_{m}} d z_{v}=p_{V V}\left(z_{m}\right)+k_{B} T \sum_{n=0}^{\infty} \int_{\infty}^{0} \frac{\partial K_{n}}{\partial z_{m}} d z_{v} \\
& =\frac{k_{B} T}{\pi} \sum_{n=0}^{\infty} \int_{0}^{\infty} \sum_{p=e, h} \frac{\left(R_{v L}^{(p)}+R_{m v}^{(p)}\right)\left(1-R_{v m}^{(p) 2} e^{-2 k_{z m} z_{m}}\right) R_{m v}^{(p)} e^{-2 k_{z m} z_{m}}}{\left[\left(1-R_{v L}^{(p)} R_{v m}^{(p)}\right)-\left(R_{v L}^{(p)} R_{m v}^{(p)}+R_{m v}^{(p)} R_{m v}^{(p)}\right) e^{-2 k_{z m} z_{m}}\right]\left(1-R_{m v}^{(p) 2} e^{\left.-2 k_{z m} z_{m}\right)}\right.} k_{z m} k_{\rho} d k_{\rho} \\
& =\frac{k_{B} T}{\pi} \sum_{n=0}^{\infty} \int_{0}^{\infty} \sum_{p=e, h} \frac{\left(R_{m v}^{(p)}+R_{v L}^{(p)}\right) R_{m v}^{(p)} e^{-2 k_{z m} z_{m}}}{\left(1+R_{L v}^{(p)} R_{v m}^{(p)}\right)-\left(R_{v L}^{(p)} R_{m v}^{(p)}+R_{m v}^{(p)} R_{m v}^{(p)}\right) e^{-2 k_{z m} z_{m}}} k_{z m} k_{\rho} d k_{\rho} \\
& =\frac{k_{B} T}{\pi} \sum_{n=0}^{\infty}, \int_{0}^{\infty} \sum_{p=e, h} \frac{\frac{R_{m v}^{(p)}+R_{v L}^{(p)}}{1+R_{m v}^{(p)} R_{v L}^{(p)}} R_{m v}^{(p)} e^{-2 k_{z m} z_{m}}}{1-\frac{R_{m v}^{(p)}+R_{v L}^{(p)}}{1+R_{m v}^{(p)} R_{v L}^{(p)}} R_{m v}^{(p)} e^{-2 k_{z m} z_{m}}} k_{z m} k_{\rho} d k_{\rho} \\
& =\frac{k_{B} T}{\pi} \sum_{n=0}^{\infty} \int_{0}^{\infty} \sum_{p=e, h} \frac{R_{m L}^{(p)} R_{m v}^{(p)} e^{-2 k_{z m} z_{m}}}{1-R_{m L}^{(p)} R_{m v}^{(p)} e^{-2 k_{z m} z_{m}}} k_{z m} k_{\rho} d k_{\rho}
\end{aligned}
$$

where we have used the following property of reflection coefficients:

$$
\frac{R_{m v}^{(e)}+R_{v L}^{(e)}}{1+R_{m v}^{(e)} R_{v L}^{(e)}}=\frac{\left(\frac{k_{z m}}{\mu_{m}}-\frac{k_{z v}}{\mu_{v}}\right)\left(\frac{k_{z v}}{\mu_{v}}+\frac{k_{z L}}{\mu_{L}}\right)+\left(\frac{k_{z m}}{\mu_{m}}+\frac{k_{z v}}{\mu_{v}}\right)\left(\frac{k_{z v}}{\mu_{v}}-\frac{k_{z L}}{\mu_{L}}\right)}{\left(\frac{k_{z m}}{\mu_{m}}+\frac{k_{z v}}{\mu_{v}}\right)\left(\frac{k_{z v}}{\mu_{v}}+\frac{k_{z L}}{\mu_{L}}\right)+\left(\frac{k_{z m}}{\mu_{m}}-\frac{k_{z v}}{\mu_{v}}\right)\left(\frac{k_{z v}}{\mu_{v}}-\frac{k_{z L}}{\mu_{L}}\right)}=\frac{\left.\frac{k_{z m}}{\mu_{v}}-\frac{k_{z L}}{\mu_{L}}\right)}{\frac{k_{z v}}{\mu_{v}}\left(\frac{k_{z m}}{\mu_{m}}+\frac{k_{z L}}{\mu_{L}}\right)}=\frac{\frac{k_{z m}}{\mu_{m}}}{\frac{k_{z m}}{\mu_{m}}+\frac{k_{z L}}{\mu_{L}}}=R_{m L}^{(e)},
$$

Similarly, $\frac{R_{m v}^{(h)}+R_{v L}^{(h)}}{1+R_{m v}^{(h)} R_{v L}^{(h)}}=R_{m L}^{(h)}$.

$$
\begin{aligned}
p_{L R}\left(z_{m}\right) & =p_{V R}\left(z_{m}\right)+\int_{\infty}^{0} \frac{\partial T_{z z}^{\mathrm{avg}}}{\partial z_{m}} d z_{v}=p_{V R}\left(z_{m}\right)+k_{B} T \sum_{n=0}^{\infty} \lim _{\delta \rightarrow 0} \int_{\infty}^{\delta} \frac{\partial K_{n}}{\partial z_{m}} d z_{v} \\
& =\frac{k_{B} T}{\pi} \sum_{n=0}^{\infty} \int_{0}^{\infty} \sum_{p=e, h} \frac{\left(R_{v L}^{(p)}+R_{m v}^{(p)}\right)\left(1+R_{v m}^{(p)} R_{m R}^{(p)} e^{-2 k_{z m} z_{m}}\right) R_{m R}^{(p)} e^{-2 k_{z m} z_{m}}}{\left[\left(1-R_{v L}^{(p)} R_{v m}^{(p)}\right)-\left(R_{v L}^{(p)} R_{m R}^{(p)}+R_{m v}^{(p)} R_{m R}^{(p)}\right) e^{-2 k_{z m} z_{m}}\right]\left(1+R_{v m}^{(p)} R_{m R}^{(p)} e^{\left.-2 k_{z m} z_{m}\right)} k_{z m} k_{\rho} d k_{\rho}\right.} \\
& =\frac{k_{B} T}{\pi} \sum_{n=0}^{\infty} \int_{0}^{\infty} \sum_{p=e, h} \frac{\left(R_{v L}^{(p)}+R_{m v}^{(p)}\right) R_{m R}^{(p)} e^{-2 k_{z m} z_{m}}}{\left(1-R_{v L}^{(p)} R_{v m}^{(p)}\right)-\left(R_{v L}^{(p)} R_{m R}^{(p)}+R_{m v}^{(p)} R_{m R}^{(p)}\right) e^{-2 k_{z m} z_{m}}} k_{z m} k_{\rho} d k_{\rho}
\end{aligned}
$$




$$
\begin{aligned}
& =\frac{k_{B} T}{\pi} \sum_{n=0}^{\infty} \int_{0}^{\infty} \sum_{p=e, h} \frac{\frac{R_{v L}^{(p)}+R_{m v}^{(p)}}{1+R_{v L}^{(p)} R_{m v}^{(p)}} R_{m R}^{(p)} e^{-2 k_{z m} z_{m}}}{\frac{1+R_{v L}^{(p)} R_{m v}^{(p)}}{1+R_{v L}^{(p)} R_{m v}^{(p)}}-\frac{R_{v L}^{(p)}+R_{m v}^{(p)}}{1+R_{v L}^{(p)} R_{m v}^{(p)}} R_{m R}^{(p)} e^{-2 k_{z m} z_{m}}} k_{z m} k_{\rho} d k_{\rho} \\
& =\frac{k_{B} T}{\pi} \sum_{n=0}^{\infty}, \int_{0}^{\infty} \sum_{p=e, h} \frac{R_{m L}^{(p)} R_{m R}^{(p)} e^{-2 k_{z m} z_{m}}}{1-R_{m L}^{(p)} R_{m R}^{(p)} e^{-2 k_{z m} z_{m}}} k_{z m} k_{\rho} d k_{\rho} .
\end{aligned}
$$

\section{APPENDIX C: DERIVATION FOR EQ. (16)}

$$
\begin{aligned}
p_{L R}\left(z_{m}\right) & =\frac{k_{B} T}{\pi} \sum_{n=0}^{\infty} \int_{0}^{\infty} \sum_{p=e, h} \frac{R_{m L}^{(p)} R_{m R}^{(p)} e^{-2 k_{z m} z_{m}}}{1-R_{m L}^{(p)} R_{m R}^{(p)} e^{-2 k_{z m} z_{m}}} k_{z m} k_{\rho} d k_{\rho} \\
& =\frac{k_{B} T}{\pi} \sum_{n=0}^{\infty} \int_{\frac{\xi_{n}}{c} \sqrt{\varepsilon_{m} \mu_{m}}}^{\infty} \sum_{p=e, h} \frac{R_{m L}^{(p)} R_{m R}^{(p)} e^{-2 k_{z m} z_{m}}}{1-R_{m L}^{(p)} R_{m R}^{(p)} e^{-2 k_{z m} z_{m}}} k_{z m}^{2} d k_{z m} \\
& =\frac{k_{B} T}{\pi} \sum_{n=0}^{\infty}, \frac{\xi_{n}^{3}}{c^{3}}\left(\varepsilon_{m} \mu_{m}\right)^{3 / 2} \int_{1}^{\infty} \sum_{p=e, h} \frac{R_{m L}^{(p)} R_{m R}^{(p)} e^{-2 k_{z m} z_{m}}}{1-R_{m L}^{(p)} R_{m R}^{(p)} e^{-2 k_{z m} z_{m}} \frac{k_{z m}^{2}}{c_{n}^{2}} \varepsilon_{m} \mu_{m}} \frac{d k_{z m}}{\xi_{n}} \sqrt{\varepsilon_{m} \mu_{m}} \\
& =\frac{k_{B} T}{\pi} \sum_{n=0}^{\infty}, \frac{\xi_{n}^{3}}{c^{3}}\left(\varepsilon_{m} \mu_{m}\right)^{3 / 2} \int_{1}^{\infty} \sum_{p=e, h} \frac{R_{m L}^{(p)} R_{m R}^{(p)} e^{-2 q \xi_{n} \sqrt{\varepsilon_{m} \mu_{m}} z_{m} / c}}{1-R_{m L}^{(p)} R_{m R}^{(p)} e^{-2 q \xi_{n} \sqrt{\varepsilon_{m} \mu_{m}} z_{m} / c}} q^{2} d q \\
& =\frac{k_{B} T}{\pi c^{3}} \sum_{n=0}^{\infty}\left(\varepsilon_{m} \mu_{m}\right)^{3 / 2} \xi_{n}^{3} \int_{1}^{\infty} \sum_{p=e, h}\left(R_{m L}^{(p)-1} R_{m R}^{(p)-1} e^{2 q \xi_{n} \sqrt{\varepsilon_{m} \mu_{m}} z_{m} / c}-1\right)^{-1} q^{2} d q
\end{aligned}
$$

where $q=k_{z m} /\left(\xi_{n} \sqrt{\varepsilon_{m}\left(i \xi_{n}\right) \mu_{m}\left(i \xi_{n}\right)} / c\right)$.

[1] H. C. Hamaker, Physica 4, 1058 (1937).

[2] E. M. Lifshitz, Sov. Phys. JETP 2, 73 (1956).

[3] S. M. Rytov, Theory of Electric Fluctuations and Thermal Radiation (Air Force Cambridge Research Center, Bedford, MA, 1959).

[4] L. D. Landau, E. M. Lifshitz, and L. P. Pitaevskii, Electrodynamics of Continuous Media (Pergamon Press, 1984).

[5] I. E. Dzyaloshinskii, E. M. Lifshitz, and L. P. Pitaevskii, Adv. Phys. 10, 165 (1961).

[6] L. P. Pitaevskii, Phys. Rev. A 73, 47801 (2006).

[7] B. W. Ninham, V. A. Parsegian, and G. H. Weiss, J. Stat. Phys. 2, 323 (1970).

[8] Y. S. Barash and V. L. Ginzburg, Sov. Phys. JETP Lett. 15, 403 (1972).

[9] Y. S. Barash and V. L. Ginzburg, Sov. Phys. Usp. 18, 305 (1975).
[10] J. Schwinger, J. L. L. DeRaad, and K. A. Milton, Ann. Phys. (NY) 115, 1 (1978).

[11] S. J. Rahi, T. Emig, N. Graham, R. L. Jaffe, and M. Kardar, Phys. Rev. D 80, 85021 (2009).

[12] A. Narayanaswamy and G. Chen, J. Quant. Spectrosc. Radiat. Transfer 111, 1877 (2010)

[13] K. Joulain, R. Carminati, J.-P. Mulet, and J.-J. Greffet, Phys. Rev. B 68, 245405 (2003).

[14] V. A. Parsegian, Van der Waals Forces: A Handbook for Biologists, Chemists, Engineers, and Physicists (Cambridge University Press, 2005).

[15] D. Gingell and V. Parsegian, J. Colloid Interface Sci. 44, 456 (1973).

[16] W. C. Chew, Waves and Fields in Inhomogeneous Media (IEEE Press, Piscataway, NJ, 1995).

[17] L. P. Pitaevskii, e-print arXiv:1011.5591. 